\title{
Sägen am eigenen Ast?
}

FMH Swiss Medical Association Herrn Präsident Dr. med. Jacques des Haller Elfenstrasse 18 3000 Bern 15

Sehr geehrter Herr Präsident Es ist grundsätzlich $\mathrm{zu}$ begrüssen, dass die Schweizerische Ärztezeitung eine liberale Anzeigenpolitik verfolgt. Doch der liberale Gedanke hat dort seine Grenzen, wo dem Verband und seinen Mitgliedern Schaden zugefügt wird.

Dies war in der Ausgabe Nr. 14 vom 5. April 2006 der Fall, in welcher ein Inserat der CSS abgedruckt wurde, in der diese eine Informationsveranstaltung für die Patientenbehandlung im Ausland anpries. Die Veröffentlichung dieses Inserates ist aus dreierlei Gründen bedenklich:

Erstens dient das Inserat dazu, ein nicht gesetzeskonformes Vorgehen der Krankenkassen zu sanktionieren. Das nach wie vor gültige und vom Schweizer Stimmvolk verabschiedete KVG verbietet den Versicherern, Leistungen für Patienten aus der Grundversicherung, die im Ausland erbracht werden, zu übernehmen. Verschiedene Kassen versuchen jedoch seit geraumer Zeit, auch Patienten aus der Grundversicherung einen Spitalaufenthalt - zurzeit im Bereich der Akutbehandlung sowie der Rehabilitation - im Ausland schmackhaft zu machen.

Zweitens schadet dieser Patiententourismus der Versicherer vor allem uns Ärzten. Denn mit der Verlegung von Patienten in Billigdestinationen wird das in der Schweiz aufgebaute medizinische und pflegerische Know-how unterlaufen und gefährdet.
Drittens widerspricht die Veröffentlichung des Inserates auch der Stellungnahme unseres Verbandes zur Vernehmlassung der Revision über die Verordnung zur Krankenversicherung KVV, mit der der Bundesrat versucht, den Gesetzesbruch verschiedener Kassen im nachhinein rechtlich $\mathrm{zu}$ sanktionieren. $\mathrm{Zu}$ Recht hatte unser Verband in seiner Stellungnahme darauf hingewiesen, dass die Behandlung Schweizer Patienten im Ausland nur zugelassen werden sollte, wenn sich im Gegenzug auch ausländische Patienten in der Schweiz behandeln lassen können. Zudem wurde in der FMH-Vernehmlassungsantwort betont, dass es nicht nur darum ginge, «billig» im Ausland einzukaufen, sondern dass auch die «Förderung des Qualitätsmedizinstandortes Schweiz ein wichtiges und berechtigtes Anliegen» sei. Mit der Politik der Krankenversicherer wird jedoch in erster Linie der Qualitätsmedizinstandort Schweiz getroffen und geschwächt.

Darunter leidet letztendlich die bisherige hochstehende medizinische Versorgung der Gesamtbevölkerung in unserem Lande. Das kann nicht im Interesse von uns Leistungserbringern sein.

Wir danken Ihnen für Ihr Interesse und grüssen Sie freundlich.

Dr. med. Hanspeter Gmünder, Medizinischer Direktor Rehaklinik Bellikon, Vorstand SW!SS REHA

Prof. Dr. med. Thierry Ettlin, Chefarzt und Medizinischer Direktor Reha Rheinfelden, Vorstand SW!SS REHA 